\title{
ESTUdO PALINOLÓgICO dE SEDIMENTOS DA BACIA DE ICó, CRETÁCEO dO ESTAdo dO CEARA, BRASIL
}

\author{
M.R.Lima ${ }^{1}$
}

\section{RESUMO}

Cincoenta e quatro espécies de pólens e esporos foram identificadas através do estudo de amostras de sondagem efetuada na Bacia de Icó, situada na parte sul do Estado do Ceará. Entre estas, a presença conjunta de Klukisporites cf. foveolatus (= Foveotriletes sp.), Exesipollenites tumulus, Inapertumopollenites turbatus e Inaperturopolienites simplex permite a inclusåo das associaçठ̄es no intervalo representativo das Zonas Palinológicas P250/P-260 do zoneamento da Petrobrás, que, em termos cronoestratigráficos, corresponde à parte média do Andar Alagoas, de idade aptiana. A julgar pelas características da palinoflora presente, os sedimentos representativos depositaram-se em ambiente continental, sob condiçōes de clima quente e úmido.

\section{ABSTRACT}

Fifty-four pollen and spore species were identified in samples from a core drilled in the Icó Basin, central southern portion of Ceará State, Brazil.

Among these species, the presence of Klukisporites cf. foveolatus $(=$ Foveotriletes sp.), Exesipollenites tumilus, Inaperturopollenites turbatus and Inaperturopollenites simplex allows the assemblages to be placed in Palynological Zones P-250/P-260 of the Petrobrás zonation, which chronostratigraphically represent the middle part of the Alagoas Stage, of Aptian age. This assignment is reinforced by the general characteristics of the palynofloras, typical of this chronologic interval, which are also suggestive of a nonmarine depositional environment under hot and wet climatic conditions.

\section{INTRODUÇĀo}

A Bacia de Icó, situada na região sudeste do Estado do Ceará, representa uma das várias ocorrências de sedimentos cretáceos continentais do Nordeste do Brasil. Em geral, é referida na literatura dentro de uma abordagem mais ampla, na qual aparece como integrante de um conjunto que engloba, de oeste para leste, as Bacias de Iguatu, Cabeça de Negro, LimaCampos e Icó (Fig. 1). Neste posicionamento, é implícita a idéia, aceita pela maioria dos autores, de que todas estas áreas são apenas fragmentos, preservados pelo tectonismo, de uma capa sedimentar antes contínua, que se poderla estender, para leste, através do Estado da Paraíba, unindo-se à Bacia do Rio do Peixe.

1Departamento de Paleontologia e Estratigrafia, Instituto de Geociências/USP, Săo Paulo. 


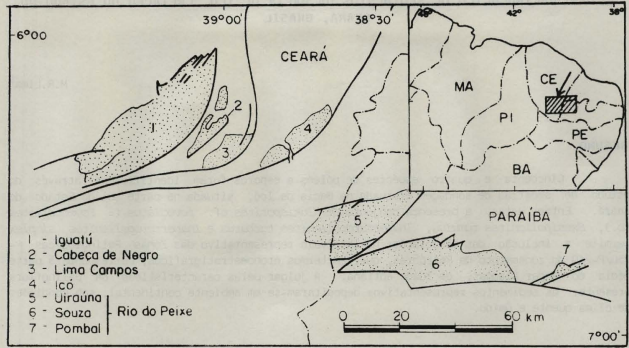

Figura 1 - Bacias cretáceas Interiores dos Estados do Ceará e Paraíba (adaptada de SCHOBBE NHAUS FILHO, 1974).

0 único estudo efetuado, de forma isolada, sobre esta bacia, corresponde a uma coletânea de textos inéditos, apresentados como Relatórios de Graduação na Escola de Geología da Universidade Federal de Pernambuco, em 1961, por Heronides Dias de Barros, Everaldo Z.V. de Mello, Maurício Cardoso do Rego e José Adriano Coelho. D desconhecimento acerca de sua geologia é praticamente total, limitando-se os diversos autores que a têm mencionado, a considerar sua sequência sedimentar como um equivalente reduzído dos Grupos Río do Peixe ou Iguatu, propostos para as bacias homônimas.

Neste contexto, o trabalho ora apresentado representa un estudo pioneiro năo só no aspecto palinológico mas também paleontológico, já que as únicas mençōes à presença de fósseis sao referências sucintas, efetuadas pelos autores citados, sendo muito precário o tratamento sistemático das formas encontradas. O objetivo, além da identificação dos palinomorfos presentes, é de datar os sedimentos portadores, contribuindo deste modo para a obtenção de dados útels nos estudos que venham a ser futuramente desenvolvidos na área.

\section{BACIA DE ICÓ}

A bacia $e$ representada por uma faixa sedimentar fuslforme, de direçăo SW-NE, estruturalmente de padrăo monoclinal, possuindo cerca de $50 \mathrm{~km}$ de comprimento por $8 \mathrm{~km}$ de largura máxima. E parcialmente atravessada pelo rio Salgado e riachos tributários que, pela geraçåo de extensos depósitos quaternários, mascaram, em alguns locais, seus 1 imites precisos. 
De qualquer modo, é patente o seu condicionamento tectônico, encaixada que está entre falhamentos, mais claramente visiveis na sua borda sul, alguns dos quais correspondendo a importantes traços tectônicos do embasamento da regiăo, como a Falha de Jaguaré. Os sedimentos exibem algumas evide̊ncias de condicionamento tectônico. BARROS (1961) ilustra, no mapa apresentado em anexo, várias medidas de mergulhos, algumas das quais expressivas $\left(40^{\circ}\right)$, a grande maioria orientada em díreça ao sistema de falhas do bloco sul. GASPARY (1967) menciona a presença de janelas do embasamento dentro da área sedimentar, o que sería sugestivo de pequena espessura da sequência sedimentar, especialmente em comparaçăo com a das bacias de Iguatu e Rio do Peixe. Esta chegou a ser estimada por CAMPOS et al. (1979) em cerca de $570 \mathrm{~m}$.

0 preenchimento sedimentar da Bacia de Icó inicia-se por sedimentos imaturos, mal selecionados, que incluem brechas e conglomerados brechóides. Gradualmente, estes passam a arenitos grosseiros ou conglomeráticos, arcosianos, cremes ou avermelhados. Acima desta sequência, ocorrem arenitos finos, menos arcosianos, com acamamento fíno, micáceos, com intercalações de leitos finos de argilito e siltito. A parte superior da seção é considerada monótona e essencialmente pelítica. Caracteriza-se por uma sucessão de camadas argilosas com acamamento horizontal e arenitos finos, raramente mais grosseiros. CAMPOS et al. (1979) mencionam a ocorrência de importantes afloramentos da parte inferior da seçăo, que alcançaria cerca de $390 \mathrm{~m}$ de espessura da bacla, na sua extremidade norte, ao longo da rodovia BR-116. Os mesmos autores atríbuem uma espessura de cerca de $180 \mathrm{~m}$ para a parte superior, que estaria bem representada na região central da bacia.

Do ponto de vista formal, há divergências entre os autores com relaçăo à sequência sedimentar acima descrita. GASPARY (1967) propōe que os sedimentos da Bacia de Icó fariam parte da "Série" Iguatu, correspondendo aos termos mais superiores das seis sequências por ele individualizadas $\left(\mathrm{Ki}^{1}\right.$ a $\left.\mathrm{Ki}^{6}\right)$. CRUZ \& FRANÇA (1971) também admitem correlaçăo com a Bacia de Iguatu, embora não indiquem qual a parte da seção exposta em Icó. MABESOONE \& CAMPANHA (1973/1974) concordam com os autores precedentes no que se refere à identidade dos sedimentos das bacias de Icó e Iguatu, embora admítindo a semelhança com a Sequência da Bacia do Río do Peixe. Propōem os autores que as seis sequências estabelecidas por GASPARY (1967) sejam englobadas em três formaçбes (da base para o topo: Quixoá, Malhada Vermelha e Lima Campos), constantes do Grupo Iguatu. A Formação Lima Campos seria, segundo os autores, a única presente na Bacia de Icó. Finalmente, CAMPOS et al. (1979) consideram que a sequência sedimentar presente corresponde integralmente à da Bacia do Rio do Peixe, propondo as mesmas designaçð̄es (Formaçð̃es Antenor Navarro e Sousa) para os pacotes inferior e superior descritos para a Bacia de Icó.

\section{DADOS PALINOLÓGICOS}

\section{Proveniência das amostras}

As amostras analisadas palinologicamente procedem da sondagem intitulada Poço Catavento, efetuada pela CONESP (Companhia Nordestina de Perfuraçōes). A sondagen, com 70 m de profundidade, localiza-se na extremidade norte da Bacia (Fig. 2). Os sedimentos amostrados correspondem a arenitos finos, cinzentos, algo silticos, que caracterizam toda a seçăo perfurada.

Oito amostras foram estudadas, representando os intervalos, do topo para a base, de $15-18 \mathrm{~m}, \quad 18-21 \mathrm{~m}, 21-24 \mathrm{~m}, 24-27 \mathrm{~m}, 27-30 \mathrm{~m}, 30-33 \mathrm{~m}, \quad 33-36 \mathrm{~m}$ e $36-39 \mathrm{~m}$. 0 material foi processado para análise segundo técnica palinológica padrăo. De cada amostra foram estudadas duas låminas, que encontram-se depositadas na coleção cientifica do DPE/IG-USP sob os números GP/4T-156 a GP/4T-171. 

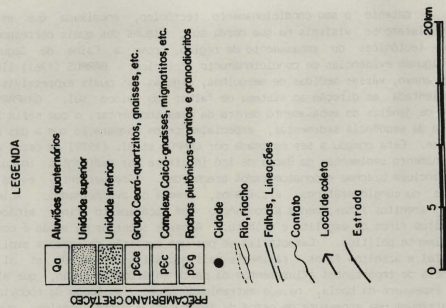

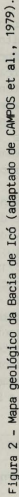

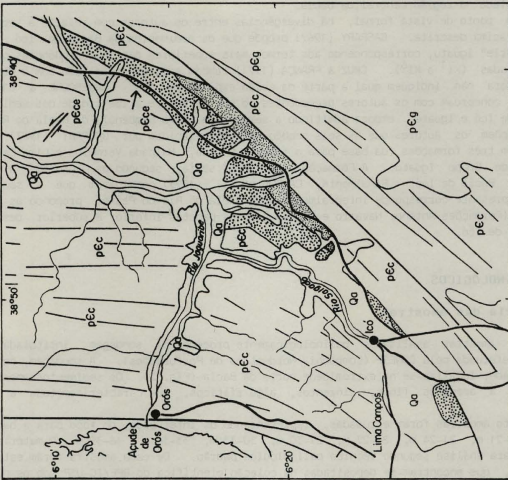




\section{RESULTADOS OBTIDOS}

Cincoenta e quatro espécies foram identificadas, tendo por base, entre outros, os trabalhos de BRLDONI \& ARCHANGELSKY (1983), BALME (1957), BRENNER (1968), BURGER (1966), COUPER (1958), DETTMANN (1963), DERHEFER (1977), GROOT \& GROOT (1962), JARDINE \& MAQOIRE (1965), LIMA (1978), NILSSON (1958) PADEN PHILLIPS \& FELIX (1971), POCOCK (1964), REGALI et al. (1974) e REYRE (1973). A lista completa é a seguinte:

\section{Esporos}

Stereisparites psilatus (Ross) Manum.

Deltoidospora nana Burger

Deltoidospora hallii Miner

Deltoidospora junctum (Kara Murza) Singh

Biretisporites potoniaei Delcourt \& Sprumont

Cyathidites minor Couper

Concavisporites jurienensis Balme

Concavisporites cf. limatulus Paden Phillips \& Felix

Concavissimisporites punctatus (Delcourt \& Sprumont) Brenner

Concavissimisporites verrucosus (Delcourt \& Sprumont) Delcourt et al.

Concavissimisporites sp.

Auritulinasporites deltaformis Burger

Obtusisporis concavus Pocock

cf. Bolchovitinaesporites congregatus (Bolkhovitina) Semenova

Leptolepidites psarosus Norris

Leptolepidites verrucatus Couper

Leptolepidites major Couper

Verrucosisporites sp.

Grarulatisporites sp.

Staplinisporites caminus (Balme) Pocock

Acanthotriletes varispinosus Pocock

Pilosisporites crassiangulatus (Ivanova) Dorhofer

Cicatricasisporites microstriatus Jardiné \& Magloire

Cicatricosisporites brevilaesuratus Brenner

Cicatricosisporites subrotumdus Brenner

Cicatricosisporites hallei Delcourt \& Sprumont

Cicatricosisporites tersus Kara Murza

Cicatricosisporites mediostriatus (Bolkhovitina) Pocock

Cicatricosisporites cuneiformis Pocock

Klukisporites of. foveolatus Pocock

Reticulatisporites arcuatus Brenner

Lycopodiumsporites rosewoodensis (de Jersey) Burger

Impardecispora of. uralensis (Bolkhovitina) Dev

Trilobosporites canadensis Pocock

Trilabosporites cf. bemissartensis (Delcourt \& Sprumont) Potonié

Appendicisporites matesovae (Bolkhovitina) Norris

Appendicisporites potamacensis Brenner

Appendicisporites sellingii Pocock

Appendicisporites of. erdtmanii Pocock

Marattisporites scabratus Couper 


\section{Polens}

Spheripollenites psilatus Couper

Inaperturopollenites turbatus Balme

Inaperturopollenites simplex Regali et al.

Exesipollenites tumulus Balme

Perinopollenites elatoides Couper

Araucariacites australis Cookson

Classopollis torosus (Reissinger) Couper

Classopollis classoides pflug

Zonallapol lenites dampieri Balme

Cycadopites nitidus (Balme) de Jersey

Cycadopites fragilis Singh

Cycadopites sp.

Monosulcites subgranulosus Couper

Eucamiidites troedssonif Dettmann \& Hughes

\section{DISCUSSÃo}

\section{Idade}

Até o momento, a Idade dos sedimentos da Bacia de Icó é incerta. Na verdade, a maioria dos autores (MORAES, 1924; BEURLEN, 1964; GASPARY, 1967; DANTAS, 1974; MABESOONE \& CAMPANHA, 1973/1974; CAMPOS et al., 1979, entre outros) concorda que a maior parte, ou mesmo a totalidade dos sedimentos representativos dos Grupos Rio do Peixe/Iguatu (e consequentemente os da Bacia de Icó) seja de idade cretácea inferior. A base para esta atribuiçăo tem sido fornecida por uma documentação paleontológica bastante escassa, constituída por ostracodes, conchostráceos, restos (principalmente escamas) de peixes e pegadas de répteis, melhor estudadas na Bacia do Rio do Peixe. 0 exato posicionamento destes níveis, contudo, dentro do Cretáceo Inferior ainda năo foì, até o momento, referído.

A associação polínica estudada permite, con segurança, a dataçăo dos níveís portadores. Entre as cincoenta e quatro espécies identificadas, a presença conjunta de Klukisporites cf. foveolatus (= Foveotriletes sp. in REGALI et al., 1974), Exesipollenites tumulus, Inaperturopollenites turbatus e Inaperturopollenites simplex torna possivel a inclusăo da associaçăo no intervalo representativo das Zonas Palinológicas P-250/P-260 do zoneamento da Petrobrás. Estas săo, em termos cronoestratigráficos, representativas da parte média do Andar Alagoas, cuja idade é o Aptiano.

A presença de sedimentos desta idade seria, até certo ponto, previsível, já que o intervalo cronológico referido parece ser o mais bem representado nas bacias interiores do nordeste brasileiro. Duas questర̄es merecem, contudo, uma atençăo especial na interpretaçăo do significado desta ocorrência: a posição dos níveis estudados em relaçăo à coluna estratigráfica da bacia e a posição desta com relaçăo à presença nas bacias do Río do Peixe e Iguatu, cujo preenchimento é muito mais espesso.

Com relaçăo ao prímeiro aspecto, pouco pode ser acrescentado. 0 caráter dos sedimentos estudados (siltitos e arenitos finos, argilosos, cinza-esverdeados) é semelhante ao dos que ocorrem em vários pontos da BR-116 na parte norte da bacia. Toda esta faixa de afloramentos é posicionada por CAMPOS et al. (1979) como pertencente à parte inferior da seçăo, cuja descriçăo torna aceitável que o intervalo ora estudado corresponda à sua parte superior, única porção onde ocorrem leitos finos de silito e argilito. 
No que diz respeito ao segundo ponto, os dados são ainda mais precários, pois são desconhecidos, até o momento, horizontes ou fósseis-guia que permitam correlaçớes seguras entre as bacias. CAMPOS et al. (op. cit.) consideram todo o pacote inferior da Bacia de Icó como equivalente à Formação Antenor Navarro, unidade basal da Bacia do Rio do Peixe. Esta correlaçăo parece insustentável, uma vez que dados de LIMA \& COELHO (1987) demonstram, também palinologicamente, que as trés unidades estratigráficas presentes nesta bacia săo mais antigas, correspondendo ao Andar Aratu (Cretáceo basal). Por outro lado, como já foi mencionado, GASPARY (1967) e MABESOONE \& CAMPANHA (1973/1974) consideram que os sedimentos da Bacia de Icó seriam equivalentes aos termos maís superfores presentes na Bacia de Iguatu (pacote $k^{6}$ de Gaspary ou Formação Lima Campos de Mabesoone \& Campanha). Este ponto de vista parece mais defensável, embora implique no fato de que a maior parte do preenchimento sedimentar desta bacia, sendo o topo de idade aptiana, seria necessariamente mais antigo. A presença de ostreídeos na Bacia de Cabeça de Negro (MELO, 1964) seria um argumento contrário a este ponto de vista, já que estes animais deveriam estar relacionados, mesmo de forma Indireta, à ingressర̃es marinhas registradas na região, iniciadas, como é sabido, no final do Aptiano, sendo portanto sugestivas da presença de sedimentos mais jovens na área.

Em sintese, a dataçăo obtida é válída tão somente para cerca de $25 \mathrm{~m}$ de sedimentos en posiçăo não muito bem definida na sequência da bacia, năo estando portanto excluída a possibilidade da presença de niveis mais velhos ou mais novos. Com respeito ao posicionamento da sequência da Bacia de Icó com relaçăo as demaís, ao que parece, havería correspondência com a parte superior do Grupo Iguatu, embora não exatamente com o seu topo.

\section{Ambiente}

A total ausência de elementos do paleomicroplâncton marinho atesta condiçōes continentais de deposição, o que é perfeitamente compatível com o quadro geral de evolução paleogeográfica da região. A abundância de esporos é ainda indicativa de clíma quente e úmido na época de deposição dos sedimentos. Em certos niveis (21-24 m, 30-33 m) algumas formas encontradas representam provavelmente acritarcas devonianos. Sua má preservação, contudo, não permitiu conclusరes definitivas, ficando apenas registrada a possibilidade da existência de sedimentos desta idade na regiăo, tal como ocorre nas bacias próximas de Jatobá, Mirandiba, Maranhão e Plataforma Continental do Ceará.

\section{AGRADECIMENTOS}

Ficam aqui registrados nossos agradecimentos aos Drs. Alarico Frota de Albuquerque Mont 'Alverne ( 42 Distrito do DNPM) e Sylvio Péricles de Oliveira (CONESP) pela cessão das amostras estudadas. Agradecimentos são também devidos aos Profs. Thomas R. Fairchild, Juracy B.0. Vespucci e Setembrino Petri pelas críticas e sugestøes, bem como ao Prof. João Cipriano de Freitas (Centros Integrados de Ensino Superior Farias Brito) pela mobilização de funcionários, que permitiu em pouco tempo a datilografia do trabalho. 


\section{REFERENCIAS BIBLIOGRAFICAS}

BALDONI, A. \& ARCHANGELSKY, S. (1983) Palinologia de la Formación Springhill (Cretacico Inferior) subsuelo de Argentina y Chile Austral. Revista Española de Micropaleontología, 15(1):47-101.

BALME, B.E. (1957) Spore and pollen grains from Mesozoic of Western Australia. C.S.I.R.O.: Austr.Coal.Res., 25:1-54.

BARROS, H.D. (1961) Geologia da faixa leste de Bebedouro-Icó, Ceará. Relatório de Graduação. Recife, Universidade Federal de Pernambuco, 28 .

BEURLEN, K. (1964) Introdução à estratigrafia geral e comparada. Recife, Expansão Gráfica. $440 p$.

BRENNER, G.J. (1968) Middle cretaceous spores and pollen from Northeastern Peru. Pollen et Spores, 10(2):341-384.

BURGER, D. (1966) Palynology of Uppermost Jurassic and Lowermost cretaceous strata in the eastern Netherlands. Leidse Geologische Mededelingen, 35:209-276.

CAMPOS, M.; BRAGA, A.P.G.; MELLO, A.A.; SOUZA, E.M.; SILVA, F.A.F.; FRANÇA, J.B. (1979) Projeto Rio Jaguaribe. Relatório Final de Ceologia. Brasilia, MME/DNPM. p.1-149 (Série Geología 4, Secăo Geología Básica, 1).

COUPER, R.A. (1958) British Mesozoic microspores and pollen grains. Paleontographica, $1038(4 / 6): 75-179$.

CRUZ, W.B. \& FRANÇA, H.P.M. (1970) Inventário hidrogeológico do Nordeste; folha 14. Jaguaribe-SO. Texto explicativo. Série Hidrogeologia, 31:1-222.

DANTAS, J.R.A.; CORDANI, U.G.; SCHOBBENHAUS FILHO, C. (1974) Carta Geológica do Brasil ao millionésimo. Fôlha Jaguaribe (SB-24), Fôlha Fortaleza (SA-24). Texto explicativo. Brasillia, DNPM. 95p.

DETTMANN, M.E. (1963) Upper Mesozoic microfloras from Southeastern Australia. Proceedings Royal Society of Victoria, $77(1): 1-148$.

DQRHOFER, G. (1977) Palynologie und stratigraphie der Buckerberg formation (BerriasiumValanginium) in der Hilsmulde (NW-Deutschland). Geologisches Jahrbuch. Reihe A. (42):1122.

GASPARY, J. (1967) Estudo geral de base do Vale do Jaguaribe. Grupo de estudos do Vale do Jaguaribe. Hidrogeologia, $7: 1-245$.

GROOT, J.J. \& GROOT, C.R. (1962) Plant microfossils from Aptian, Albian and Cenomanian deposits of Portugal. Comunicações Serviços Geológícos Portugal, 46: 133-176.

JARDINE, S. \& MAG.OIRE, L. (1965) Palynologie et stratigraphie des bassins du Senegal et de Côte d'Ivoire. Mémoires du Bureau de Recherches Geologiques et Minières, BRGM, (32): 187245. 
LIMA, M.R. (1978) Palinologia da Formaçăo Santana (Cretáceo do Nordeste do Brasil). Såo Paulo, 335p. (Tese de Doutorado, Instituto de Geociências/USP).

LIMA, M.R. \& COEL.HO, M.P.C.A. (1987) Estudo palínológico da sondagem estratigráfica de Lagoa do Forno, Bacia do Rio do Paixe, Cretáceo do Nordeste do Brasil. Boletím IG-USP, Série Cientifica, 18:67-83.

MABESOONE, J.M. \& CAMPANHA, V.A. (1973/1974) Caracterizaçăo estratigráfica dos Grupos Rio do Peixe e Iguatu. Estudos Sedimentológicos, $(3 / 4): 22-41$.

MELO, P.G. (1964) Geologia da faixa de Igaroi-Icó, Ceará. Arquivos de Geología, 5:57-80.

MORAES, L.J. (1924) Serras e montanhas do Nordeste. Publicaçăo Inspectoria de Obras Contra as Seccas, $58(2): 1-120$.

NILSSON, T. (1958) Wer das Vorkommen eines Mesozoischen Sapropelgesteins in Schonen. Lunds Universitets Arsskrift, 54(10):3-111.

PADEN PHILLIPS, P. \& FELIX, C.J. (1971) A study of Lower and Middle Cretaceous spores and pollen grains from the South-eastern United States. I. Spores. Pollen et Spores, $13(2): 279-348$.

Pocock, S.A. (1964) Pollen and Spores of Chlamidospermaceae and Schizeaceae from Upper Manville strata of the Saskatoon area of Saskatchewan. Grana Palynologica, 5(2):129-209.

REGALI, M.S.P.; UESUGUI, N.; SANTOS, A.S. (1974) Palinologia dos sedimentos meso-cenazóicos do Brasil. Boletim Técnico da PETROBRAS, 17(3):177-190.

REYRE, Y. (1973) Palynologie du Mesozoique Sahariéri. Menoires Museum National d'Histoire Naturelle. Ser.C., 27:1-284. 

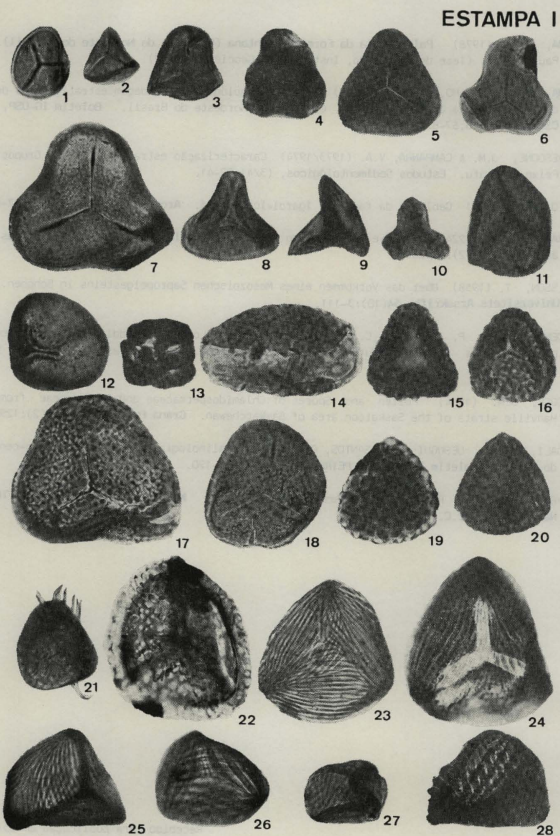

$20 \mu \mathrm{m}$ 
Bol.IG-USP, Sér.Cient., 21:35-46, 1990
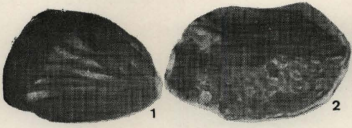

ESTAMPA II
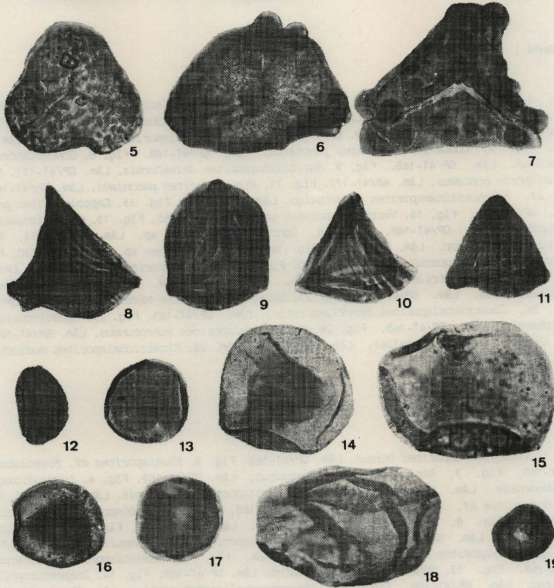

15
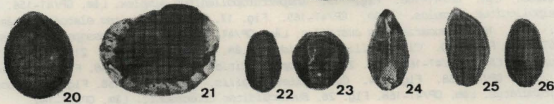

$20 \mu \mathrm{m}$ 


\section{LEGENDAS DAS ESTAMPAS}

Todas as figuras aumentadas $600 x$

\section{ESTAMPA I}

Fig. 1. Stereisporites psilatus. Làm. GP/4T-169. Fig. 2. Deltoidaspora nana. Lâm, GP/4T-165. Fig. 3. Deltoidospora hallii. Lâm. GP/4T-156. Fig. 4. Deltoidospora junctum. Låm. GP/4T-164. Fig. 5. Cyathidites minor. Lâm. GP/4T-168. Fig. 6. Cancavisporites jurienensis. Låm. GP/4T165. Fig. 7. Concavissimisporites punctatus. Lâm. GP/4T-168. Fig. 8. Concavisporites cf. limatulus. Lâm. GP/4T-168. Fig. 9. Auritulinasporites deltaformis. Låm. GP/4T-171. Fig. 10. abtusisporis concavus. Lâm. 68/4T-171. Fig. 11. Biretisporites potaniaei. Lâm. GP/4T-165. Fig. 12. cf. Bolchovitinaesporites congregatus. Lâm. GP/4T-170. Fig. 13. Leptolepidites psarosus. Làm. GP/4T-165. Fig. 14. Verrucosisporites sp. Lâm. GP/4T-163. Fig. 15. Concavissimisporites verrucosus. Lâm. GP/4T-169. Fig. 16. Concavissimisporites sp. Lâm. GP/4T-171. Fig. 17. Leptolepidites major. Làm. GP/4T-168. Fig. 18. Granulatispoorites sp. Lâm. GP/4T-170. Fig. 19. Leptolepidites verrucatus. Låm. GP/4T-170. Fig. 20. Staplinisporites caminus, Lâm. GP/4T-168. Fig. 21. Acanthotriletes varispinosus. Låm. GP/4T-169. Fig. 22. Pilosisporites crassiangulatus. Làm. GP/4T-169. Fig. 23. Cicatricosisporites microstriatus. Lâm, GP/4T-171. Fig. 24. Cicatricosisporites brevilaesuratus. Lam. GP/4T-165. Fig. 25. Cicatricosisporites cuneiformis. Lâm, GP/4T-165. Fig. 26. Cicatricosisporites subrotundus. Lâm, GP/4T-169. Fig. 27. Cicatrioosisporites hallei. Lâm. GP/4T-157. Lâm. 28. Cicatricosisporites mediastriatus. Lam. GP/4T-168.

\section{ESTAMPA II}

Fig. 1. Cicatricosisporites tersus. Lâm. GP/4T-168. Fig. 2. Klukisporites cf. foveolatus. Låm. GP/4T-163. Fig. 3. Reticulatisporites arcuatus. Lam. GP/4T-169. Fig. 4. Lyoopodiumsporites rosewoodensis. Lâm. GP/4T-170. Fig. 5. Impardecispora cf. uralensis. Làm. GP/4T-165. Fig. 6. Trilabosporites cf. bemissartensis. Lâm. GP/4T-168. Fig. 7. Trilobosporites canadensis. Lâm. GP/4T-168. Fig. 8, Appendicisporites matesovae. Lam, GP/4T-169. Fig. 9. Appendicisporites potomacensis. Låm. GP/4T-164. Fig. 10. Appendicisporites cf. enditmanii. Lâm. GP/4T-171. Fig. 11. Appendicisporites sellingii. Lâm, GP/4T-164. Fig. 12, Marattisporites scabratus. Làm. GP/4T-168. Fig. 13. Spheripollenites psilatus. Lâm. GP/4T-168. Fig. 14. Inaperturopollenites turbatus. Lâm. GP/4T-168. Fig. 15. Inaperturopollenites simplex. Lâm. GP/4T-156. Fig. 16. Exesipollenites tumilus. Lâm. GP/4T-165. Fig. 17. Ferinopollenites elatoides, Låm. GP/4T164. Fig. 18. Araucariacites australis. Lâm. GP/4T-168. Fig. 19. Classopollis torosus. Lâm. GP/4T-165. Fig. 20. Classopollis classoides. Làm. GP/4T-156. Fig. 21, Zonallapollenites damoieri, Lâm. GP/4T-169. Fig. 22. Cycadopites nitidus. Låm. GP/4T-168. Fig. 23. Cycadopites sp. Lâm. GP/4T-168. Fig. 24. Cycadiopites fragilis. Låm, GP/4T-158. Fig. 25. Nonnsulcites: subgranulatus. Lâm. GP/4T-168. Fig. 26. Eucomiidites troedssonii. Låm. GP/4T-164. 